\title{
20. SPIRAL STRUCTURE OF THE GALAXY DERIVED FROM THE HAT CREEK SURVEY OF NEUTRAL HYDROGEN
}

\author{
H. WEAVER \\ Radio Astronomy Laboratory, University of California, Berkeley, Calif., U.S.A.
}

\begin{abstract}
The extensive Hat Creek survey of neutral hydrogen combined with southern observations provides the basis for a new discussion of the spiral structure of the galaxy. The purpose of this investigation is to provide a general picture of the galaxy. It is found that the pitch of the spiral arms is approximately $12^{\circ} .5$ and that there are many spurs and interarm features as we observe in external galaxies.

The sun is not located in a major spiral arm, but rather in a spur or offshoot originating near or at the Sagittarius arm, which is a major structure in the galaxy. The young stars in the general vicinity of the sun delineate this spur, not a major arm structure. The stars and the gas are in agreement in indicating a large pitch angle $\left(20^{\circ}-25^{\circ}\right)$ for this local structure, which differs from the smaller pitch angle for the arms which form the system as a whole.

In the presentation a computer-produced movie of the galaxy based on Hat Creek hydrogen contour maps similar to those in Figure 1 was shown. It was used to illustrate generally the complexity of the gas structure and, in particular, to show (i) observational aspects of the spur in which the sun is located and (ii) the point of origin of the so-called Perseus arm.
\end{abstract}

\section{The Hat Creek Survey of Neutral Hydrogen}

The recently automated 85-foot telescope at the Hat Creek Observatory has been used in conjunction with the 100-channel receiver to complete a neutral-hydrogen survey in the region of the galactic plane (Weaver and Williams, 1969). At each half-degree interval throughout the longitude range $10^{\circ}$ to $250^{\circ}$ a uniformly-spaced sequence of 81 neutral-hydrogen profiles was observed over the latitude range $-10^{\circ}$ to $+10^{\circ}$. The observations comprising the survey are thus spaced by 0.25 (one-half beam width) in latitude and by 0.5 (one beam width) in longitude. The data from the 501 observed latitude cuts are displayed in the form of computer-produced contour maps of which samples are shown in Figure 1. The instrumental and statistical data that characterize the observations are brought together in Table I.

TABLE I

Observational data relating to Hat Creek survey of neutral hydrogen

Sky coverage:

Frequency coverage: Frequency resolution: Data display:

Integration time: RMS fluctuation: Angular resolution:
Every $0^{\circ} .25$ in galactic latitude between limits $-10^{\circ}$ to $+10^{\circ}$; every $0^{\circ} .5$ in galactic longitude over range $10^{\circ}$ to $250^{\circ}$ (19521 profiles) Each profile covers $250 \mathrm{~km} \mathrm{~s}^{-1} ; 200$ information points per profile $10 \mathrm{kHz}\left(2.11 \mathrm{~km} \mathrm{~s}^{-1}\right)$ spaced every $5 \mathrm{kHz}$

Contour maps as shown in Figure 1. Contour intervals $T_{\mathrm{A}}=1^{\circ}, 2^{\circ}$, $4^{\circ}, 8^{\circ}, 15^{\circ}, 20^{\circ}, 30^{\circ}, \ldots$

$72 \mathrm{sec}$ per profile

$0.18 \mathrm{~K}$ (system noise $\leqslant 150 \mathrm{~K}$ )

$35^{\prime} .5$ HPBW 

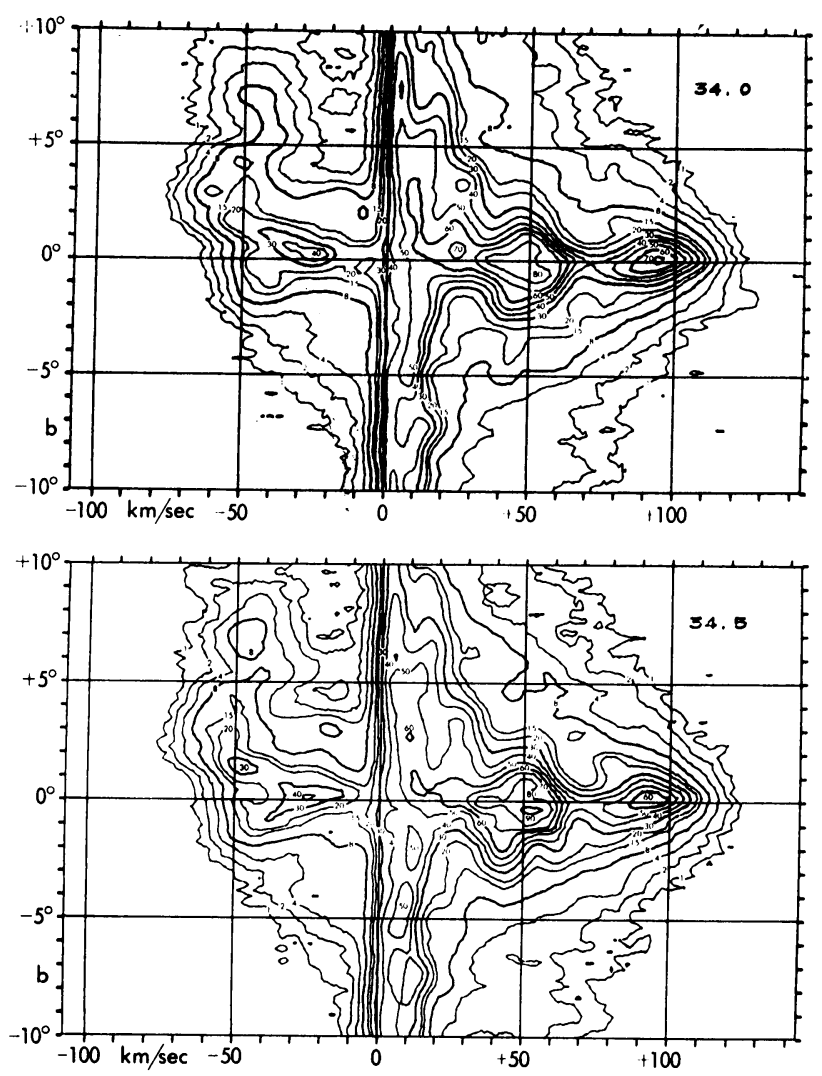

Fig. 1. Sample of a page from the Hat Creek survey of neutral hydrogen.

Completion of this extensive survey makes possible a new discussion of the spiral structure in the galaxy.

\section{A Method of Deriving Spiral Structure from Observations of Neutral Hydrogen}

To illustrate the method of analysis to be used in determining spiral structure, we take as a mathematical model of the galaxy the smooth, continuous spiral shown in Figure $2 \mathrm{a}$. We assume that over the $R$-range of interest ( 3 to, say, $15 \mathrm{kpc}$ ) the rotation curve of the model galaxy is a continuous monotonic function $\omega(R)$ of $R$ alone. Under these conditions the spiral in Figure $2 \mathrm{a}$, if observed from the sun shown in Figure 2a at galactocentric distance $R_{0}$, would produce in the observational longitude, radial velocity $\left(l^{\mathrm{II}}, v\right)$ coordinate system the characteristic pattern of loops and sinelike curves shown in Figure 2b. Points on the inner part of the spiral, for which $R<R_{0}$, transform to points in quadrants II and IV of Figure $2 \mathrm{~b}$ and characteristically lie along loops. Points on the outer parts of the spiral $\left(R>R_{0}\right)$ transform to points 
in quadrants I and III of Figure $2 \mathrm{~b}$ and form sine-like curves. At those points at which the spiral crosses $R=R_{0}$ (we move along the spiral locus so that $R$ increases) the corresponding $l^{\mathrm{II}}, v$ locus crosses from quadrant II to III or IV to I, and thus may prevent the completion of a full loop in quadrants II or IV. It may readily be shown that all complete loops in quadrants II and IV in Figure $2 \mathrm{~b}$ are tangent to the envelope curve $v=R_{0}\left[\omega\left(R_{0} \sin l^{\text {II }}\right)-\omega\left(R_{0}\right)\right] \sin l^{\text {II }}$, from which the rotation curve $\omega(R)$ is found observationally.
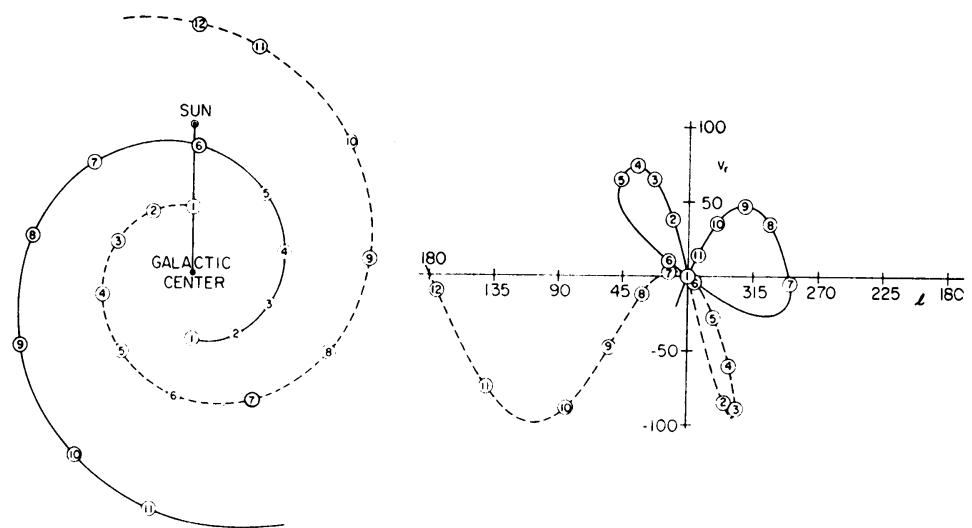

Fig. 2. (a) (left) Model spiral galaxy. - (b) (right) longitude-velocity $(l \mathrm{II}, v)$ diagram for the spiral shown on the left. Corresponding points in the spiral and its $\left(l^{\mathrm{II}}, v\right)$ diagram may readily be identified ( $l$ means $l$ II $)$.

The pattern shown in Figure $2 \mathrm{~b}$ is typical; the precise form and phase of any $l^{\mathrm{II}}, v$ locus is determined, of course, by the form and phase of the generating spiral, $R(\theta)$, and by the rotation curve, $\omega(R)$.

Real galaxies (see the Hubble Atlas of Galaxies, Sandage, 1961) are more complex than a pair of smooth spirals differing in phase by $180^{\circ}$. Real galaxies consist, characteristically, of spiral arms (often broken into segments which may differ in pitch and phase) plus interarm links, spurs, and other less regular features. If we investigate a slightly more realistic galaxy with such features as are illustrated in Figure $3 \mathrm{a}$, we still obtain in the observational $l^{\mathrm{II}}, b^{\mathrm{II}}$ coordinate system a diagram typically like that of Figure $2 b$, except that it contains, additionally, bits and pieces of curves which are the $l^{\text {II }}, v$ transformations of the interarm links, spurs, segments and so forth, pictured in Figure 3a.

The general approach in this discussion of spiral structure will be to construct the observationally determinable $l^{\mathrm{II}}, v$ diagram and from that to infer the nature and form of the spiral galaxy.

\section{Construction of the $l^{\text {II }}, v$ Diagram}

The concentrations of hydrogen which form the gas arms in the galaxy are of finite cross section. Investigations which we cannot describe in detail here show that within 


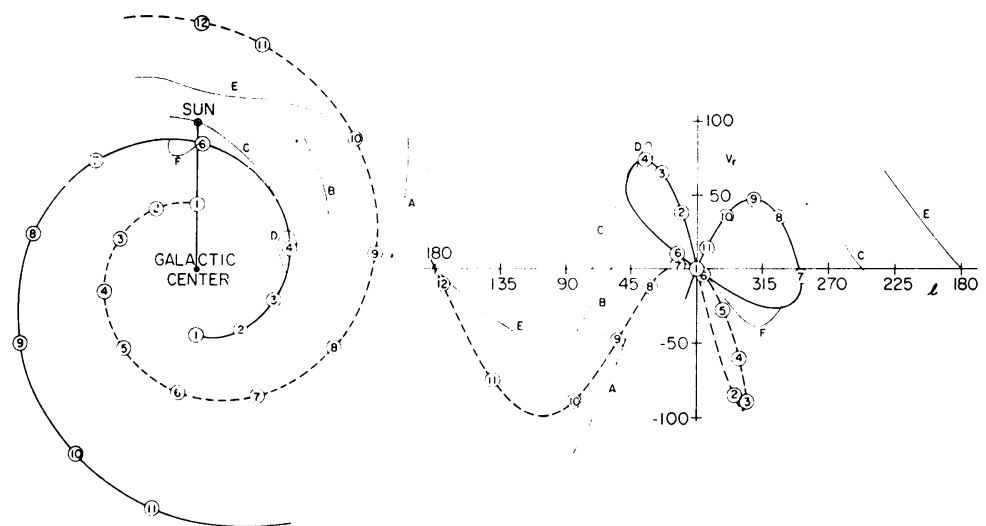

Fig. 3. (a) (left) Model spiral galaxy with sample spurs, interarm, and other features; - (b) (right) longitude-velocity $\left(l^{\mathrm{II}}, v\right)$ diagram for the structures shown on the left.

a gas arm there are many subunits or subconcentrations which show continuity over significant ranges of longitude. As a working model, we imagine the arm to be made up of subunits or subconcentrations drawn out along the arm. Characteristically, then, the cross section of the arm at a given $\theta$ value would show gas concentrations at slightly different $R$ and $z$ (height above the plane) values. Observing from the sun, we would see these subconcentrations within the arm at slightly different latitudes, $b^{\mathbf{I I}}$, and velocities, $v$, at some given longitude, $l^{\text {II }}$. On each observed contour map, which, specifically is a $b^{\mathrm{II}}, v$ map for a given $l^{\mathrm{II}}$, we measure the velocity of each of the subconcentrations within an arm or arm-like structure. In the present investigation in which the goal is to produce an overall picture of the galaxy, we ignore minor latitude differences within an arm structure and treat all subconcentrations within an arm structure similarly, projecting them onto the galactic plane.

To construct the $l^{\text {II }}, v$ diagram we require for our analysis, we plot each measured velocity as a function of longitude; to show relatedness of subconcentrations we connect with a vertical line all those points representing subconcentrations within an arm or arm-like structure.

In the observed $l^{\mathrm{II}}, v$ diagram, Figure 4 , the points in the longitude range $10^{\circ}$ to $230^{\circ}$ are from the Hat Creek Survey; those in the longitude range 300 to 360 are from the observations by Kerr (1969); those indicated by open triangles in the longitude range 290 to 310 are from Varsavsky (1969), and the points shown as filled triangles in the longitude range 280 to 300 are $\mathrm{H}$ II velocities from Wilson (1969). These latter have been added to strengthen the diagram in an important longitude range in which adequate $\mathrm{HI}$ observations are currently unavailable.

\section{The Pitch of the Spiral Arms}

If a galaxy has generally smooth continuous arms as shown in Figure 5a, we can provide a general characterization of the spiral structure by specifying the pitch angle 


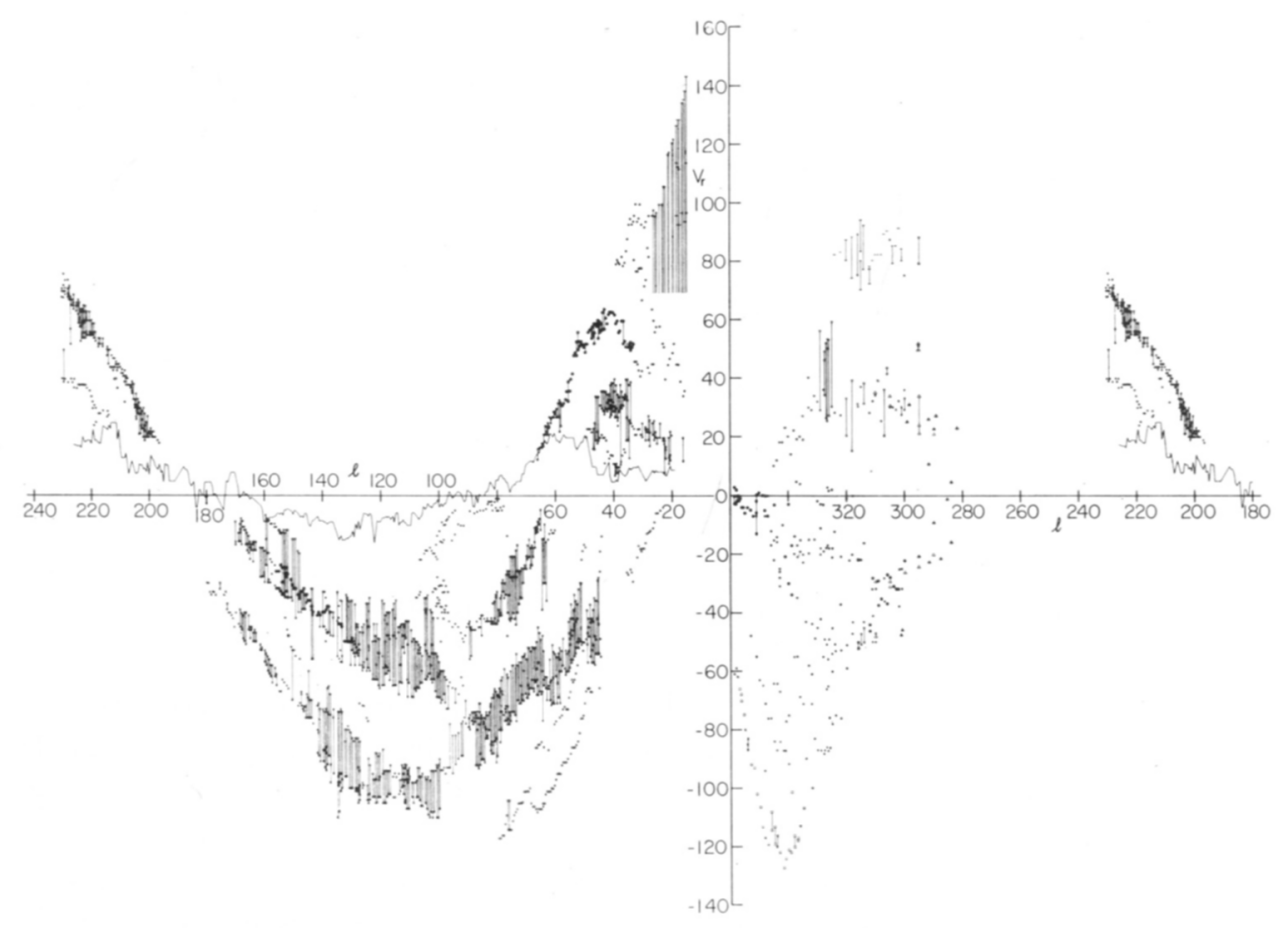

Fig. 4. Observed $l^{\mathrm{II}}, v$ diagram for the galaxy. Intensity of features is indicated by size of symbol. See Section 4 for detailed expansion of data sources. The open squares represent points on the expanding $3 \mathrm{kpc}$ arm.

(a)

(b)
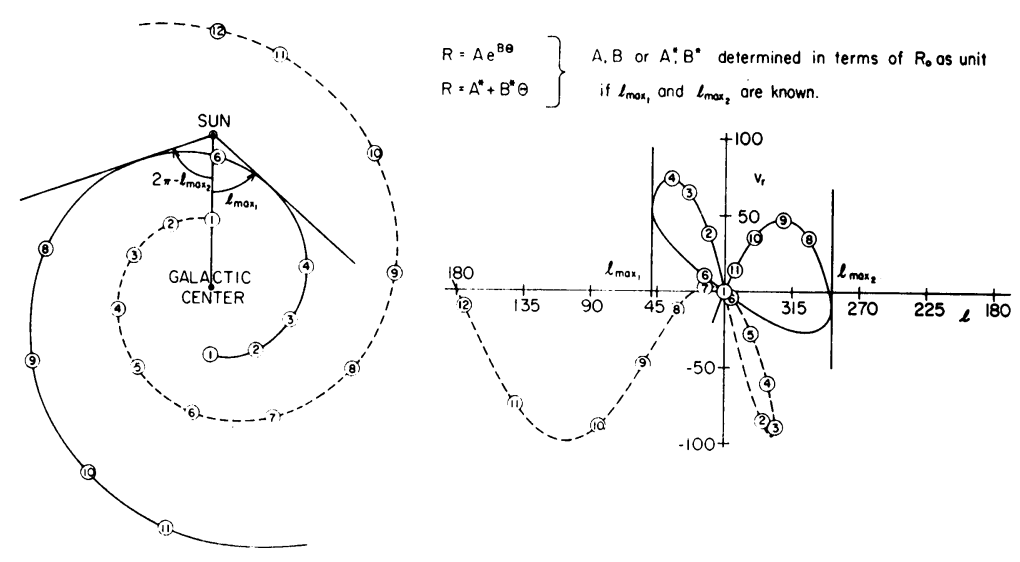

Fig. 5. Diagram illustrating the tangents $l_{\max 1}$ and $l_{\max 2}$ (see text). 
of the spiral. For a galaxy such as that shown in Figure 5a there exist well-defined tangents to the arms. Figure $5 \mathrm{~b}$ displays the observationally determinable $l^{\mathrm{II}}, v$ diagram corresponding to the spiral in Figure 5a. The longitudes $l_{\max 1}$ and $l_{\max 2}$ at which tangency occurs can be found from observation as illustrated in Figure $5 \mathrm{~b}$. In turn, knowing $l_{\max 1}$ and $l_{\max 2}$, one may employ numerical methods to find values of the parameters $A, B$ or $A^{*}, B^{*}$ (defined in Figure 5) which characterize the arm of the galaxy contained within the longitude range between the tangents. For the galaxy we

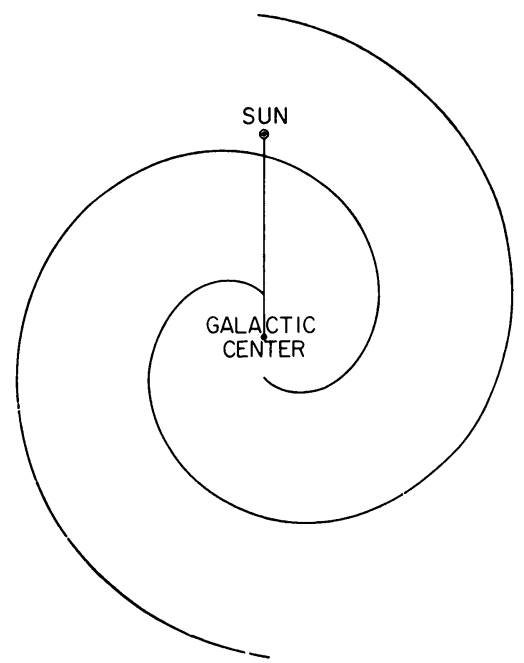

Fig. 6. A smoothed galactic model inferred from the $\left(A^{*}, B^{*}\right)$ values derived from observed $l_{\max 1}$ and $l_{\max 2}$ values.

find $l_{\max 1}=50^{\circ} .5, l_{\max 2}=284.0$ (details of the determination are given elsewhere by Weaver, 1969), and from these values. $A=0.410, B=0.245 ; A^{*}=0.202, B^{*}=0.220$. The pitch angle of the arm is 12.5 in the solar vicinity. The overall form of the galaxy deduced from the $\left(A^{*}, B^{*}\right)$ values is shown in Figure 6.

\section{A More Detailed Picture of the Galaxy}

The galaxy is more complex than indicated by Figure 6 ; there are more features in the galactic $l^{\text {II }}, v$ diagram than can be accounted for by the spirals in Figure 6 . To obtain a more detailed picture of the spiral structure in the system, we represent the arm and arm-like features of the $l^{\mathrm{II}}, v$ diagram by segments of curves as illustrated in Figure 7. With the Schmidt (1965) rotation curve, we transform these segments in the $l^{\text {II }}, v$ plane to the $R, \theta$ plane, and obtain the picture shown in Figure 8 .

The two sides of the galaxy appear rather different in character in Figure 8 . The difference is not physically real; it is caused by the scarcity of observations from the southern sky. The southern data cover only the latitude range $+2^{\circ}$ to $-2^{\circ}$ at intervals of $1^{\circ}$ in longitude. While it is evident from these observations that there are many 


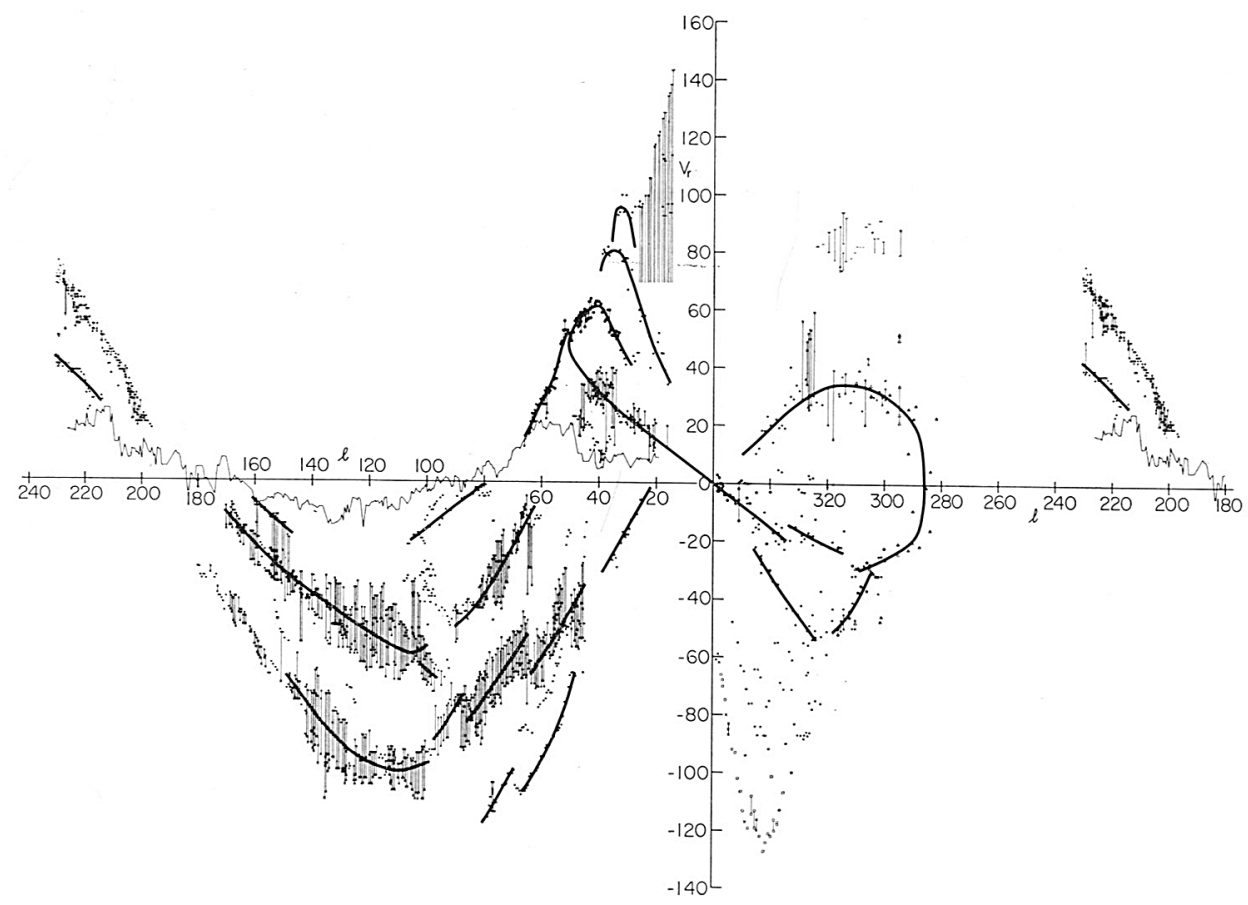

Fig. 7. Representation of $\left(l^{\mathrm{II}}, v\right)$ features.

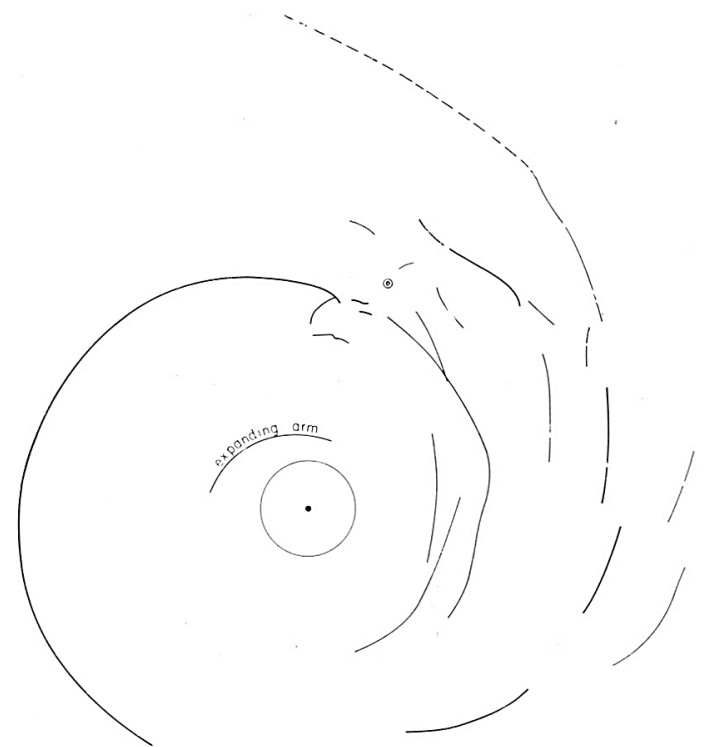

Fig. 8. Observational picture of the galaxy derived by transforming the loci in Figure 7 to $R$, $\theta$ through the rotation curve. The lack of detail on the left-hand side of the diagram is observational only and reflects no real aspect of the galaxy (see text). 
details in the southern structure, those details often cannot be traced with complete certainty; they have therefore not been drawn in Figure 7 or Figure 8. For the northernsky section of Figure 8 the data used in the analysis cover the latitude range $-10^{\circ}$ to $+10^{\circ}$ at every 0.5 in longitude. Such extensive sky coverage makes the tracing of spiral structure much more certain.

In Figure 8 the structure on the right-hand side of the spiral should be taken as more characteristic of the galaxy. On the left-hand side of the spiral only gross features have been depicted; many other spiral features are present in this part of the diagram, but they have not been entered in the picture because of lack of precise information.

The spiral shown in Figure 8 resembles a late $\mathrm{Sb}$ or an Sc galaxy that might well be broadly characterized by the smooth spiral shown in Figure 6. It contains many spurs and inter-arm fragments of the type visible on pictures of galaxies.

\section{The Local Hydrogen Structure: Model Calculations}

That the sun is located in an arm-like structure is clearly indicated by many observations; hydrogen is seen everywhere around us. However, the sun is not in a major arm, but rather in an offshoot or spur of the Sagittarius arm.

A variety of radio observations indicate the orientation of the feature in which the sun is located. The $21-\mathrm{cm}$ continuum map by Westerhout (1958) shows the Cygnus complex at $l^{\mathrm{II}} \sim 80^{\circ}$ as a clearly separate entity. The southern $21-\mathrm{cm}$ continuum map by Mathewson et al. (1961) shows the Vela complex at $l^{\mathrm{II}} \sim 264^{\circ}$ as a separate unit similar in angular size to Cygnus. These two complexes, separated by very nearly $180^{\circ}$ in longitude, are the result of our looking fore and aft along the arm-like structure in which the sun is located.

How such a structure, seen from within, will reveal itself in the hydrogen line radiation is indicated in Figures 9, 10, and 11 on the basis of a model (Figure 9) in which we show the sun inside a spiral structure. At such a location, gas surrounds

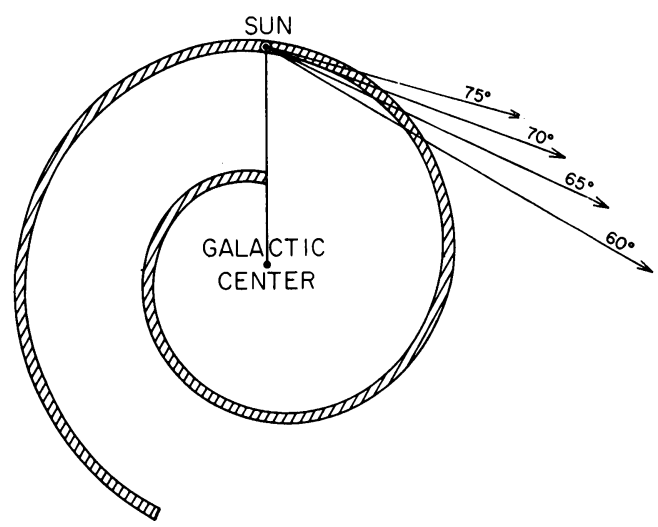

Fig. 9. A model spiral gas structure containing the sun. Lines of sight at $l \mathrm{II}=60^{\circ}, 65^{\circ}, \ldots$ are illustrated. 
the sun in every direction. Lines of sight are shown at $l^{\mathrm{II}}=60^{\circ}, 65^{\circ}, \ldots$. The line of sight at $60^{\circ}$ passes through the gas immediately surrounding the sun; it traverses the gas structure a second time at a distance of several $\mathrm{kpc}$ from the sun. The same situation is found for the line of sight at $65^{\circ}$ except that the second traversal is less distant from the sun. Finally, however, at some longitude $l_{\mathrm{c} 1}$ the line of sight no longer traverses the structure twice. At $l_{\mathrm{c} 1}$, and for $l^{\mathrm{Il}}>l_{\mathrm{c} 1}$, there is only a single traversal of the structure. A corresponding critical line of sight at longitude $l_{\mathrm{c} 2}$ exists at approximate longitude $360^{\circ}-l_{\mathrm{c} 1}$, at which there is again a change from single to double traversal. The exact numerical value of $l_{\mathrm{c} 2}$ depends upon the form (particularly upon the curvature and width) of the gas structure.
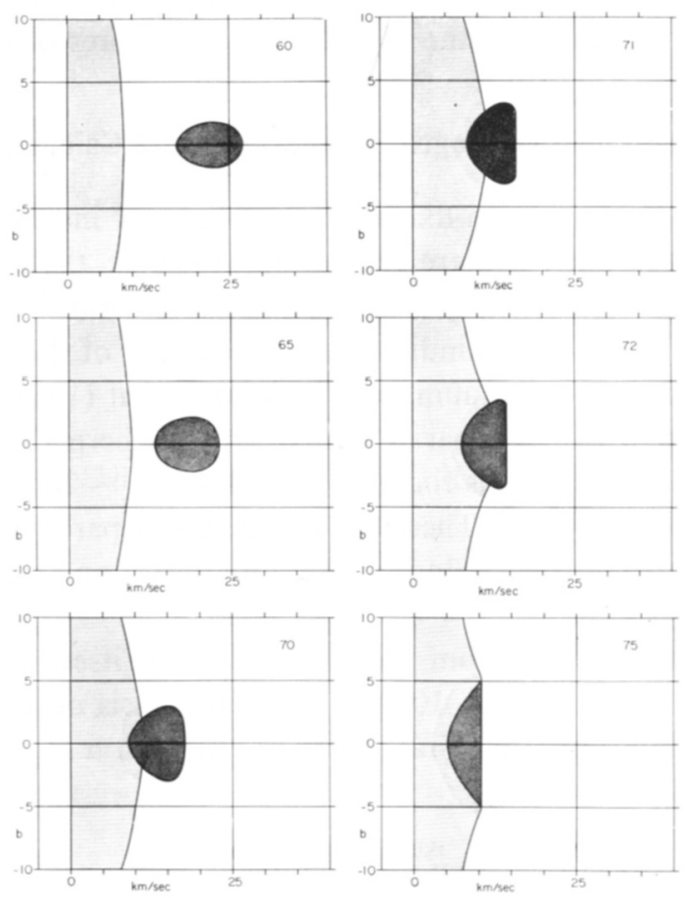

Fig. 10. Theoretical $b^{11}, v$ contour diagrams (shown in outline form) computed for the model shown in Figure 9. The series is especially designed to show the merging of the second-traversal feature and the surrounding gas (see text).

The critical longitudes $l_{\mathrm{c} 1}$ and $l_{\mathrm{c} 2}$ divide the longitude circle into two sectors. In the inner sector extending from $l_{\mathrm{c} 2}$ to $l_{\mathrm{c} 1}$ and containing $l^{\mathrm{II}}=0^{\circ}$, a line of sight traverses the structure twice. (This will be termed the two-traversal sector.) In the outer sector extending from $l_{\mathrm{c} 1}$ to $l_{\mathrm{c} 2}$ and containing $l^{\mathrm{II}}=180^{\circ}$, the line of sight traverses the structure only once. (This will be termed the one-traversal sector.) We omit consideration of special cases in which curvature of the gas structure is such that one or both critical $l$-values cease to exist or become multiple.

To delineate in detail the form of the gas structure in which the sun is located, 
we must specify three distances $d_{1}, d_{2}, d_{3}$ for each longitude in the two-traversal sector, and one distance $d_{1}$ at each longitude in the one-traversal sector. Specifically,

$d_{1}=$ the distance from the sun to the point at which the line of sight leaves the gas structure;

$d_{2}=$ the distance from the sun to the point at which the line of sight re-enters the gas structure;

$d_{3}=$ the distance from the sun to the point at which the line of sight leaves the gas structure a second time.

In the one-traversal sector the quantities $d_{2}$ and $d_{3}$ do not exist. In the two-traversal sector as $l^{\mathrm{II}} \rightarrow l_{\mathrm{c} 1}, d_{1} \rightarrow d_{2}$. As the line of sight moves from the two-traversal to the onetraversal sector, $d_{1}$ has a discontinuity at $l_{\mathrm{c} 1}$, at which longitude $d_{1} \rightarrow d_{3}$ in numerical value.

Observationally, we determine the Doppler counterparts of $d_{1}, d_{2}, d_{3}$, not the distances themselves. Moreover, by the methods of measurement described in Section 3, we would not measure Doppler counterparts of $d_{2}$ and $d_{3}$ separately, but the Doppler counterpart of some average $\left\langle d_{2}, d_{3}\right\rangle$, the precise character of which would depend mainly upon the density distribution within the gas structure. For the purposes of this investigation, the Doppler counterpart of the average $\left\langle d_{2}, d_{3}\right\rangle$ is adequate; $\left\langle d_{2}, d_{3}\right\rangle$ defines the run of the gas structure, it only fails to provide information on the width of the gas structure, which we here do not specifically investigate.

In the case of $d_{1}$, however, an effort has been made to define the 'edge' of the gas structure, that is, an effort has been made to determine the Doppler counterpart of $d_{1}$ in the belief that $d_{1}$ will be physically more meaningful than $\left\langle 0, d_{1}\right\rangle$. Details of the observational procedure of making the determination are given elsewhere (Weaver, 1969).

In Figure 10 we show in outline theoretical $b^{\mathrm{II}}, v$ contour diagrams for the $l^{\mathrm{II}}$-values considered in the model in Figure 9. The 'surrounding' gas is of wide latitude distribution since it is nearby; it starts at $v=0$ and extends to a $v$-value corresponding to $d_{1}$ for $l^{I I} \leqslant l_{\mathrm{c} 1}$. For $l^{\mathrm{II}} \leqslant l_{\mathrm{c} 1}$ the distribution extends to $v_{\max }\left(l^{\mathrm{II}}\right)$, which is unrelated to $d_{1}$. The second feature, shown darker in the $b^{\mathrm{II}}, v$ diagrams, is of smaller latitude extent than the surrounding gas; it arises from the line of sight's second traversal of the gas structure. For $l^{\mathrm{II}}=60^{\circ}$, the feature representing the second traversal in the model appears at $\langle v\rangle=22 \mathrm{~km} \mathrm{~s}^{-1}$. As $l^{\mathrm{II}} \rightarrow l_{\mathrm{c} 1}$ the feature representing the second traversal (i) moves in toward the 'surrounding' gas, finally overlapping it, and (ii) increases in extent in latitude since the average distance $\left\langle d_{2}, d_{3}\right\rangle$ decreases. Finally, at longitude $l_{\mathrm{c} 1}$ the separate feature arising from the second traversal merges; there is a single distribution starting at $v=0$ and extending to $v_{\max }\left(l^{11}\right)$. There may be folding in the latter distribution, however, in that gas from two points on the line of sight may have the same velocity. Such double points, like the separate feature, are shown dark in Figure 10.

Figure 11 displays the theoretical $l^{\text {II }}, v$ diagram corresponding to the model in Figure 9 and the theoretical $b^{\mathrm{II}}, v$ maps in Figure 10. In accord with the convention of Section 3, the distant, second-traversal feature is represented by mean-value points 
(the Doppler counterparts of $\left\langle d_{2}, d_{3}\right\rangle$ ). The 'surrounding' gas is shown as a continuous line which represents the Doppler velocity equivalent to $d_{1}$ for $l^{\mathrm{II}} \leq l_{\mathrm{c} 1}$. The discontinuity at $l_{\mathrm{c} 1}$ occurs because a line of sight for which $l^{\mathrm{II}}>l_{\mathrm{c} 1}$ enters the singletraversal sector and there intersects the circle of maximum velocity.

The mergence of the second-traversal feature into the surrounding gas is clearly shown in Figures 10 and 11.

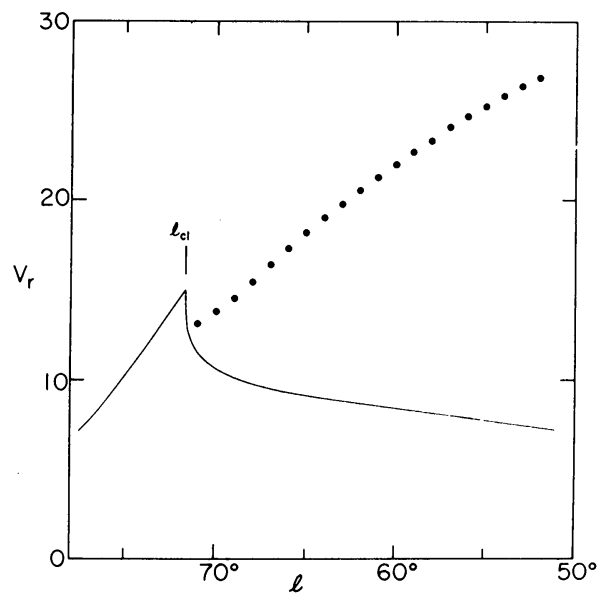

Fig. 11. Theoretical $\left(l^{\mathrm{II}}, v\right)$ diagram for the model galaxy in Figure 9 derived from $b^{\mathrm{II}}, v$ contour diagrams like those shown in Figure 10 (see text).

In the earlier galactic model displayed in Figure 3a there is a spur (Feature C) going through the sun in the same general manner as we have discussed above. The velocity-longitude counterpart of Feature $C$ is shown in Figure $3 \mathrm{~b}$. The spur in Figure 3a has no thickness and thus differs from that discussed in Figures 9, 10, and 11. As we look fore and aft along the zero-thickness spur in Figure 3a, we find that in the $l^{\text {II }}, v$ diagram Feature $\mathrm{C}$ extends to $v=0$, where it shows a discontinuity. As we look fore and aft along the spur of finite thickness, we see that there is also a discontinuity in the features arising from the second traversal by the line of sight. The secondtraversal features merge into the surrounding gas distribution in the $l^{\text {ll }}, v$ diagram, however, not the value $v=0$. The 'surrounding' or local gas is the connection between the separately-seen fore and aft sections of a spur of finite thickness that contains the sun.

\section{The Local Hydrogen Structure: Observations}

In the observed $l^{I I}, v$ diagram, Figure 4 , there are strong features in the vicinity of $l^{\mathrm{II}}=60^{\circ}$ and $l^{\mathrm{II}}=220^{\circ}$ that are analogous to Feature $\mathrm{C}$ or to the second-traversal feature in Figure 11. We note particularly that they merge into the distribution representing the surrounding gas in precisely the manner predicted by the model study and shown in Figure 11. 

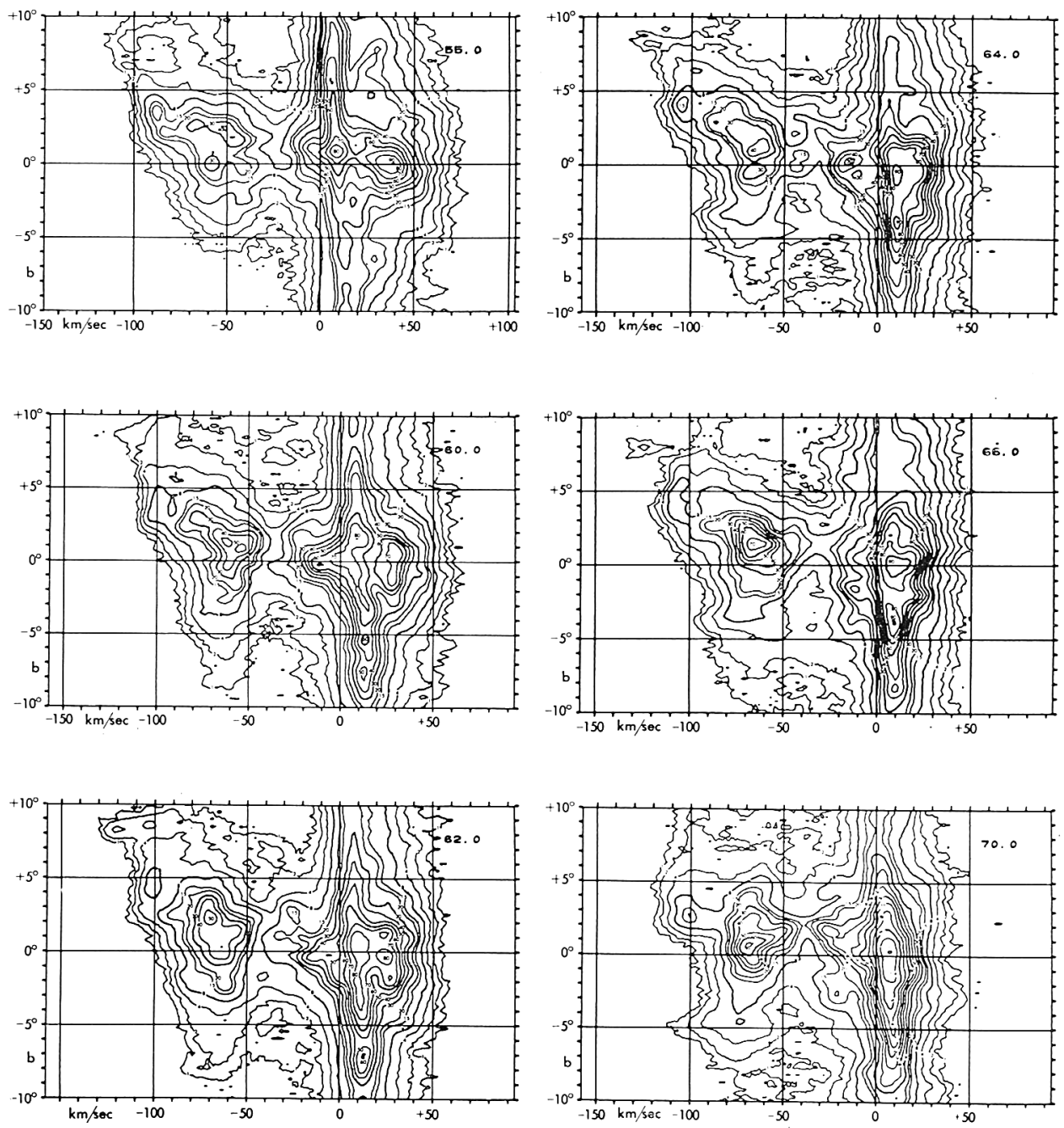

Fig. 12. Observed $b^{11}, v$ contour diagrams illustrating the behavior of the spur containing the sun. The marked similarity to the predicted (theoretical) $b^{\mathrm{II}}, v$ diagrams is striking. Note in particular how the separate feature merges into the surrounding gas.

In Figure 12 we display the observational counterpart of Figure 10. The similarity of Figures 10 and 12 is striking. In the observational diagram the feature representing the second traversal by the line of sight is at first $\left(l^{\mathrm{II}}=55\right)$ clearly separated from the surrounding gas. As $l^{\text {II }}$ increases, the separate feature increases in latitude coverage and merges with the surrounding gas precisely as predicted for a gas structure containing the sun.

In Figure 8 the structure derived by transformation of the features at $l^{\mathrm{II}} \sim 60^{\circ}$, $l^{\mathrm{II}} \sim 220^{\circ}$ in Figure 7 is shown as a spur originating at or in the vicinity of the 
Sagittarius arm. It is not shown as continuous through the sun since only the features represented by lines in Figure 7 were transformed in Figure 8. Clearly, however, the connection between the parts of the spur through the sun is provided by the surrounding gas as the model study predicts.

\section{Local Hydrogen Structure and Stars}

In Figure 13 we superimpose on Figure 8 the picture of local galactic structure defined by young stars (Schmidt-Kaler, 1964). There is no disagreement between the young stars and the gas in defining the local structure. The long-standing apparent disagreement between the pitch angle of the arms derived from the stars and that found from the gas arose because of an inappropriate comparison.

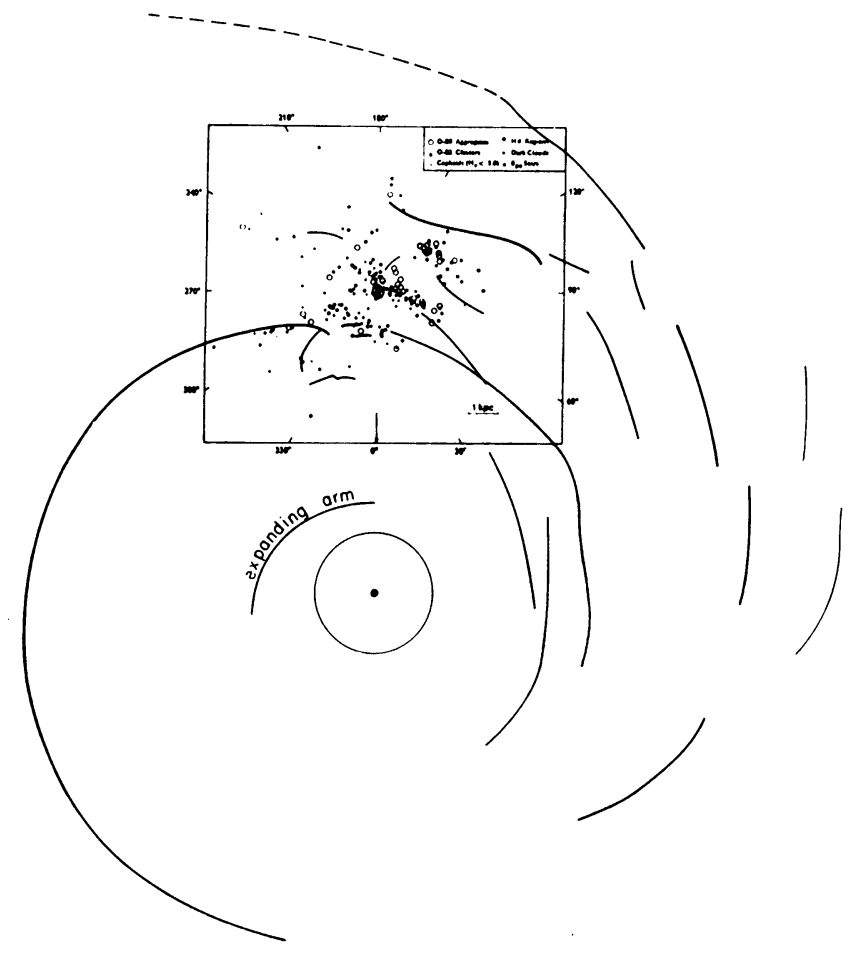

Fig. 13. The observed local distribution of young stars superimposed on the observationally determined distribution of neutral hydrogen. Each distribution was derived completely independently of the other. The agreement is remarkably good. The diagram provides an explanation of the causes of the long-standing apparent disagreement between the pitch angle derived from the (local) stars and the (galactic-scale) neutral hydrogen structure.

The observed stars are located primarily in the local spur that contains the sun. The local spur does indeed have a large pitch angle, $20^{\circ}$ to $25^{\circ}$, when defined either by stars or by gas. The pitch angle of the large-scale arm structure derived from hydrogen observations is much smaller, of the order of 12.5 . The discrepancy arose 
when the large star-derived pitch angle for the local structure was compared with the much smaller gas-derived pitch angle of the large-scale structure. There is no disagreement of pitch angle when the comparison is confined to the local structure in which the observed stars are located. There are no appropriate stellar observations to test agreement of stars and gas in defining the pitch angle on the galactic scale.

\section{References}

Kerr, F. J.: 1969, Australian. J. Phys. Astrophys. Suppl. 9, 1.

Mathewson, D. S., Healey, J. R., and Rome, J. M.: 1961, Australian. J. Phys. 15, 354.

Sandage, A.: 1961, The Hubble Atlas of Galaxies, Carnegie Institution of Washington, Washington.

Schmidt, M.: 1965, Stars and Stellar Systems 5, 528.

Schmidt-Kaler, T.: 1964, Trans.IAU 12B, 416.

Varsavsky, C.: 1969, private communication.

Weaver, H.: 1969, in preparation.

Weaver, H. and Williams, D. R. W.: 1969, in preparation.

Westerhout, G.: 1958, Bull. Astron. Inst. Netherl. 14, 215.

Wilson, T.: 1969, Ph.D. Thesis, M.I.T. 